InterSedes, Revista electrónica de las sedes regionales de la Universidad de Costa Rica, ISSN 2215-2458, Vol XXI, Número 44, Agosto - Diciembre, 2020.

10.15517/isucr.v21i44.43938 | intersedes.ucr.ac.cr | intersedes@ucr.ac.cr

\title{
SI MISMO COMO OTRO Y COMUNIDAD TERAPEÚTICA
}

IF SAME AS ANOTHER AND THERAPEUTIC COMMUNITY

Bernardo Castillo Gaitán ${ }^{1}$

\begin{tabular}{|l|l|}
\hline Recibido: 24.02 .20 & Aprobado: 29.07 .20 \\
\hline
\end{tabular}

DOI: $10.15517 /$ isucr.v21i44.43938

\section{Resumen}

Con el artículo se pretende reconocer la importancia de incorporar textos filosóficos en la fundamentación teórica de los programas de atención a personas adictas a las drogas. La introducción expone la importancia de la obra Sí Mismo como Otro en relación al tratamiento de adictos a las drogas; se presentan los fundamentos teóricos de la Comunidad Terapéutica como modelo de atención, un resumen general de obra en cuestión, continuado con la relación establecida entre la obra mencionada y la Comunidad Terapéutica. Se llega a concluir que Sí Mismo como Otro, ofrece sustento teórico para el desarrollo de programas para la atención de persona adictas a las drogas.

Palabras claves:Sí mismo; otro; adicción; tratamiento; Ricoeur.

\begin{abstract}
The article aims to recognize the importance of incorporating philosophical texts in the theoretical foundation of programs for attention to people addicted to drugs. In the introduction he exposes the importance of the work Himself as Other in relation to the treatment of drug addicts; The theoretical foundations of the Therapeutic Community are presented as a model of care, a general summary of the work in question, continued with the relationship established between the mentioned work and the Therapeutic Community. It is concluded that Himself as Other, offers theoretical support for the development of programs for the care of people addicted to drugs.
\end{abstract}

Keywords: Hinself, other, addiction, treatment, Ricoeur.

\section{Introducción}

\footnotetext{
${ }^{1}$ Costarricense. Filósofo. Profesor de Filosofía y Humanidades. Escuela de Estudios Generales. Universidad de Costa Rica, San José, Costa Rica. Email: Bernardo.castillo@ucr.ac.cr
} 
La adicción es una de las problemáticas sociales actuales más dramáticas. En el caso de Costa Rica, cada año el número, de personas que consumen drogas, sufren algún tipo de desequilibrio emocional relacionado con la adicción, va en aumento. Sin embargo el principal problema, no es el consumo de sustancias, es la persona quien tiene capacidad de decisión ante las diversas situaciones de su vida. Es en este sentido que se puede afirmar que la adicción a las drogas es un tema antropológico y del cual la antropología hermenéutica de Ricouer podría dar grandes aportes, específicamente lo desarrollado por este autor en su obra Sí Mismo como Otro

La presente investigación quiere aventurarse en el abordaje del tema de la adicción a las drogas desde la perspectiva filosófica, tomando como punto partida la obra filosófica Sí Mismo como Otro de Paul Ricoeur que se propone en su antropología hermenéutica. La forma de cómo un adicto perciben la realidad, las narraciones por medio de las cuales exponen sus experiencias de vida, ese deseo o aspiración que tienen de ser mejores y que no se logra, el deseo de construir un proyecto de vida, la tendencia de considerarse esencialmente malos y las expresiones constantes de sentir culpa y ese gran deseo externado por ellos mismo de buscar su identidad ipse, son condiciones antropológicas tratadas en la filosofía ricoueriana.

Tomando en cuenta la propuesta antropológica de Ricouer, se expone la actualidad que tiene la filosofía en la búsqueda de respuestas a situaciones dramáticas que experimenta el ser humano. "La filosofía se presenta como un proceso que tiene que ver con la vida, en la cual el yo va logrando la apropiación del sí mediante el esfuerzo por existir y de nuestro deseo de ser, a través de las obras que atestiguan ese deseo y ese esfuerzo” (Triana, 1997, p. 115).

Lo novedoso de la investigación consiste en dar un aporte teórico al programa de comunidad terapéutica que se desarrolla en el Centro de Rehabilitación al adicto Rostro de Jesús ${ }^{2}$ a partir de la antropología que Ricoeur propone en su obra Si Mimos como Otro con el fin de establecer referentes teóricos de rehabilitación de adictos a la droga.

El problema de investigación se plantea con la pregunta, ¿Qué aporta Sí Mismo como Otro de Paul Ricoeur a la comunidad terapéutica del Centro de Rehabilitación al adicto Rostro de Jesús?, ¿qué elementos teóricos de Sí Mismo como Otro se pueden incorporar al programa?, ¿cómo expresa en

\footnotetext{
${ }^{2}$ Este centro pertenece a una ONG denominada bajo el nombre jurídico Asociación Rostro de Jesús, aunque el nombre lo sugiere no es una organización religiosa, es una institución respetuosa de los Derechos Humanos de las personas, sin importar su condición social, credo religioso, preferencia sexual e ideología.
} 
la comunidad terapéutica la propuesta de Ricouer?, ¿qué enseñanzas se desprenden Sí Mismo como Otro para ayudar a los adictos a la búsqueda de su identidad?

\section{Desarrollo}

\section{Fundamentos teóricos}

En este apartado se van a exponer los dos fundamentos teóricos de la investigación. En primer lugar referente al modelo de atención al adicto denominado comunidad terapéutica, para ello se seguirá la teoría desarrollada por la directora del Centro, (Mora, 2007) y en segundo lugar, una breve exposición de la obra de antropología hermenéutica de Paul Ricouer, Sí Mismo como Otro.

\section{La comunidad terapéutica como modelo de atención al adicto}

Un programa de atención al adicto es un documento base donde se la teoría de base para la atención en el proceso de Rehabilitación, según Mora (2007, p. 18), el programa de atención denominado Comunidad Terapéutica (en adelante CT), es proyecto educativo que se basa en la rehabilitación y la reinserción social de personas con problemas de droga y de conducta, por tal razón el punto de partida no es la droga, sino la persona como protagonista de su rehabilitación. En este sentido la CT, no es un modelo impuesto, es un espacio que se brinda para ser persona.

Mora (2007), expone que el objetivo del programa de la CT es que la persona pase de su dependencia a la autonomía personal. Todo el proceso que realiza el usuario de auto-conocimiento personal y de reinserción familiar, social y laboral, le permite volver a la sociedad como un ser humano con dignidad y con identidad. Por eso la terapia más importante es la misma comunidad, donde se aprende a mejorar la autoestima y de diluye el miedo a ser rechazado por la sociedad

A través de la experiencia en la Comunidad Terapéutica, el adicto es capaz de aprender a desarrollar un estilo de vida libre de drogas. La comunidad terapéutica (CT) para el tratamiento del abuso y adicción a las drogas ha existido por alrededor de 40 años en ella se utiliza la influencia entre compañeros, mediada a través de una variedad de procesos de grupo, para ayudar a cada persona a aprender y asimilar las normas sociales y desarrollar habilidades sociales más eficaces. 
Otro principio que se presenta en una CT es la "auto-ayuda". La auto-ayuda implica que las personas en tratamiento son los principales contribuyentes al proceso de cambio. La "auto-ayuda титиа" significa que las personas también asumen una responsabilidad parcial en la recuperación de sus compañeros, un aspecto importante del propio tratamiento de la persona (Mora, 2007).

El tratamiento en la CT, tiene tres etapas básicas, a las que se le atribuye un período de tiempo como cumplimiento de días, sino como referentes que van verificando la rehabilitación del ser persona. Estas etapas son (Mora, 2007 20-21):

Etapa 1. La inducción y el tratamiento temprano: típicamente ocurren durante los primero 30 días para asimilar a la persona en la CT. El residente nuevo aprende las políticas y los procedimientos de la CT; establece una relación de confianza con el personal y los demás residentes; con ayuda inicia una evaluación personal de sí mismo, sus circunstancias y necesidades; empieza a comprender la naturaleza de la adicción; y deberá comenzar a comprometerse con el proceso de recuperación.

Etapa 2. El tratamiento primario a menudo: utiliza un modelo estructurado a través de niveles progresivos de actitudes, comportamientos y responsabilidades pro-sociales. La CT puede utilizar intervenciones para cambiar las actitudes, las percepciones y los comportamientos de la persona relacionados con el uso de drogas y dirigirse a sus necesidades sociales, educacionales, vocacionales, familiares, y sicológicas.

Etapa 3. El re-ingreso: intenta facilitar la separación de la persona de la CT y su transición exitosa a la sociedad en general. Un graduado de una CT deja el programa libre de drogas y con un empleo o habiendo regresado a la escuela. Los servicios después del tratamiento residencial pueden incluir consejería individual y familiar y asesoramiento vocacional y educativo. A menudo el tratamiento en la CT incorpora a los grupos de auto-ayuda, como Alcohólicos Anónimos o Narcóticos Anónimos, y anima a los residentes de la CT a que participen en estos grupos después del tratamiento.

\section{Sí Mismo como otro de Paul Ricoeur}

La obra de antropología hermenéutica de Paul Ricoeur, Si Mismo como Otro, es una reflexión filosófica que quiere darle un lugar a la persona entre un valor y un desprecio excesivos, 
se trata entonces de una cuestión de identidad, que se plantearía a partir de la pregunta, ¿puede mantener un adicto su identidad a pesar de su enfermedad? (Ricouer, 2003), el autor va a desarrollar una hermenéutica del sí y del otro distinto de sí, una recuperación del sujeto, que es la base de la atención al adicto en el Rostro de Jesús. Ricoeur va a plantear la recuperación del sujeto en los diez estudios.

En el primer estudio se va a referir a la persona y la referencia identificante, haciendo para ello una aproximación semántica con el fin de plantear la diferencia su pensamiento con la filosofía analítica, al respecto señala:

"Identificar algo es poder dar a conocer a los demás, dentro de una gama de cosas particulares del mismo tipo, aquella de la que tenemos intención de hablar. Precisamente en este trayecto de la referencia identificante encontramos por primera vez a la persona, en un sentido muy pobre del término, que distingue globalmente esta identidad de los cuerpos físicos. Identificar, es este estadio elemental, no es identificarse a sí mismo, sino identificar algo" (Ricouer, 2003, p.1).

El segundo estudio en la búsqueda de la identidad del sí, Ricoeur lo titula la Enunciación y el sujeto hablante. Enfoque pragmático (2003), se centra no en el enunciado, sino en la enunciación dando así importancia al sujeto que habla.

Por su lado el tercer estudio, una semántica de la acción sin agente (Ricoeur, 2003), pone de manifiesto el significado de hacer, dejando de lado el que actúa para pasar el cuarto estudio, de la acción del agente (Ricoeur, 2003), que va a dar a entender que la comprensión de la acción de alguien es como saber usar el lenguaje bajo las preguntas ¿Quién es el que hace? Y ¿quién es el que actúa? Se plantea en este punto la capacidad de lo humano.

El estudio quinto, la identidad personal y la identidad narrativa presenta el aporte propio que Ricouer (2003) da la identidad y esto es que ella es constitutiva del sí, en la definición del sí, el sujeto describe, narra y prescribe. Así pasa a abordar el sí de la identidad narrativa, tema del sexto estudio (Ricouer, 2003), se expone la idea que la ficción de una narración da unidad a las vivencias del que narra, pues la narrativa es medio entre la ipseidad y la mismidad. Para seguir dando paso a la idea que la narración da identidad, Introduce el tema de la ética en el séptimo estudio, el sí de la intencionalidad ética, se va a exponer que lo esencial del relato es lo ético pues se narra a partir del sistema de vida que se tenga. En este estudio, hace una diferencia entre la ética y la moral. “... reservaré el término ética para intencionalidad de una vida realizada, y el de moral 
para la articulación de esta intencionalidad dentro de normas caracterizadas a la vez por la pretensión de universalidad y por un efecto de restricción" (Ricoeur, 2003, p. 174).

En el octavo estudio el sí y la norma moral, el autor, va a someter la intencionalidad ética a la norma, cómo diferenciar el sí de la norma, para afirmar en el estudios nueve que la convicción es decir sí en la sabiduría práctica.

Una moral de la obligación engendra situaciones conflictivas en las que nuestro parecer, la sabiduría práctica solo posee el recurso, en el ámbito del juicio moral en situación, a la intuición inicial de la ética, a saber, el objetivo de la vida buena con y para los otros en instituciones justas. (p. 258),

Sí Mismo como Otro, cierra con el estudio décimo, ¿Hacía que Ontología? exponiendo que el ser humano tiene gran valor por su alteridad y su responsabilidad de sí sobre otro. De esta manera, la base teórica de la presente investigación, basada en Sí Mismo como Otro, servirá como referente para el desarrollo de los objetivos del mismo, pues tanto la obra de Ricouer como el programa de atención del Rostro de Jesús, buscan sacar lo mejor de sí de las personas.

\section{Relación Si Mismo como Otro con una Comunidad Terapéutica}

\section{La Comunidad Terapéutica Rostro de Jesús ${ }^{3}$}

El Rostro de Jesús es una organización no gubernamental sin fines de lucro reconocida bajo la figura jurídica de asociación con declaratoria de institución de bienestar social y de interés público. Está ubicado en el distrito Canoas del Cantón Central de Provincia de Alajuela, tiene capacidad para atender 52 pacientes, hombres en edades de 21 a 65 años, su principal característica es que es el único centro del país que es atendido por personas no adictas a las drogas.

El programa de atención al adicto de la CT Rostro de Jesús partiendo del lema "NADIE VIENE DEMASIADO PRONTO Y NINGUNO DEMASIADO TARDE", ofrece un programa residencial dirigido a hombres adictos, de 20 a 60 años de edad, de preferencia provenientes de la

\footnotetext{
${ }^{3}$ La información aquí resumida está tomada del Programa de Atención a al Adicto, aprobado y acreditado por el Instituto sobre Alcoholismo y Farmacodependencia, 2017. No está publicado
} 
provincia de Alajuela, contando para tal efecto con una planta física capaz de albergar a 53 internos y estamos en proceso de ampliación a 62 La estancia es de 6 a 18 meses, con posibilidad de realizar extensiones de hasta 6 meses, con ingreso voluntario y totalmente gratuito aunque se solicita una contribución voluntaria cuando esto es posible.

Se señala además que las adicciones degradan al individuo en muchas áreas de su vida, por lo que un buen programa de atención debe favorecer la recuperación integral del paciente, restaurando su salud física, espiritual, emocional y social. Lo anterior plantea la necesidad de ofrecer un programa dinámico y equilibrado donde el residente encuentre tranquilidad para evaluar su vida presente y plantearse un futuro mejor, facilitando un acercamiento y posterior reencuentro con la familia, el trabajo, la sociedad.

La metodología que se sigue para la atención de los pacientes es la permanente construcción de una forma particular de hacer comunidad terapéutica. ¿Por qué se dice una forma permanente y particular de construir comunidad? En el Rostro de Jesús, el problema no es la droga, sino la persona adicta. Siguiendo el concepto de problema, lo que se pone por delante para obstaculizar el desarrollo de la dignidad humana, son las mismas personas, por esto se considera que no hay una comunidad terapéutica modelo, pues, cada una responde a las necesidades, personas y coyuntura del momento histórico cultural que va definiendo la misma realidad. No se quiere decir con esto que se desconoce los pasos necesarios para formar una comunidad: número de integrante, trabajo en grupo, relación social, etc.

El lugar del desarrollo de la metodología del tratamiento, no lo constituye, solamente, un paradigma o una ideología, sino la persona misma. Este método, parte de la pregunta: ¿Cómo reconstruir la vida personal, familiar y social del interno? Todas las personas involucradas con la institución desarrollan un proceso de conocimiento en reciprocidad dialéctica asimétrica entre el internamiento y la realidad. Cada paciente es visto como signo de esperanza que permite crecer a los funcionarios y al paciente ir recuperando el Rostro de su ser íntimo. Aquí se da una pista de lo que puede aportar Sí Mismo como Otro de Paul Ricouer.

\section{Sí Mismo como Otro y Comunidad Terapéutica ${ }^{4}$}

\footnotetext{
${ }^{4}$ Comunidad Terapéutica se abrevia como CT.
} 
A partir del desarrollo del programa de la CT Rostro de Jesús se considera importante abordar los siguientes temas de Sí Mismo como Otro en relación con el programa de atención ofrecido a los adictos en proceso de rehabilitación bajo el modelo de comunidad terapéutica

\section{Concepto de Ipseidad}

El concepto de ipseidad que se propone se identifica con sí mismo, con el carácter existencial de la persona, más que de su estructura esencial, sin embargo Ricoeur, va a agregar que este concepto de identidad de sí mismo al otro, es decir, una identidad que se construye en relación con el otro que es un sí mismo. Implica la alteridad en un grado tan íntimo que no se puede pensar la una sin la otra, es decir una identidad que es alteridad. La forma de mantener, en el tiempo esta identidad como ipseidad, se realiza por medio de la palabra dada (Ricouer, 2003), es una identidad que se narra.

\section{La identidad narrativa}

La identidad narrativa, es una característica que Ricouer va a señalar para la identidad de sí mismo, "se trata de saber lo que la categoría narrativa del personaje aporta a la discusión de la identidad personal" (2003, p. 142), "la identidad de sí mismo se narra como una trama, pues la persona entendida como personaje del relato, no es una identidad distinta a sus experiencias... Es la identidad de la historia la que hace la identidad del personaje” (Ricouer, 2003, p. 147). La narración, de un acontecimiento, que se hace a otro, marca el carácter ético de la ipseidad, pues con el otro se adquiere el compromiso de ser sí mismo.

\section{La ética de la ipseidad}

Los estudios séptimo, octavo y noveno de Sí Mismo como otro, van a desarrollar lo que podría llamarse la ética de la ipseidad, se va a plantear la ética como la intencionalidad de una vida realizada, (Ricoeur, 2003). La ética de la ipseidad, se va a sintetizar en la frase tender a la vida buena en con y para otro en instituciones justas, en donde la norma moral signifique comprometerse con el otro con el fin de adquirir la convicción de llevar a la práctica la identidad de sí mismo en el otro. 


\section{Plan de Vida}

Sí Mismo como Otro, define el sentido que tiene un plan de vida expresando que se trata de configuraciones de acciones que proceden de un movimiento de vaivén entre ideales lejanos, que es preciso verificar ahora y el peso de las ventajas y de los inconvenientes de la elección de tal plan de vida en la práctica, la vida se entiende no solamente como lo biológico, sino tomando en cuenta lo existencial que abarca, también lo ético y lo cultural (Ricoeur, 2003).

\section{La recuperación del sujeto y la Comunidad Terapéutica}

La monumental obra del filósofo francés, Paul Ricoeur, Sí Mismo como Otro, tiene el mismo objetivo de una Comunidad Terapéutica que atiende adictos, esto es la recuperación del sujeto a partir de su identidad como sí mismo como otro. La necesidad del ser humano de preguntarse por su propia existencia, no es solamente teórica, es un ser que se relaciona en contexto, ya sea histórico, cultural o político en los que va a encontrar otras identidades como las propias. Se vive una tensión entre los otros, la alteridad, y uno mismo.

En relación a la recuperación del sujeto en Sí Mismo como Otro se afirma: "somos poderosos cuando comprendemos adecuadamente nuestra dependencia, en cierto modo horizontal y externa, respecto a todas las cosas y nuestra dependencia vertical e inmanente respecto al poder primordial que Spinoza también llama Dios” (Ricoeur, 2003, p. 351), quizá esta sea una de las mayores dificultades de un adicto, no reconocer a los demás para poder ser persona, esta es lo que Ricoeur y la CT plantean: recuperarse a sí mismo con y para los otros.

\section{Sí Mismo como Otro y la Asociación Rostro de Jesús}

Sí Mismo como Otro de Ricoeur, es una obra que puede dar grandes aportes a la Comunidad Terapéutica de Rostro de Jesús, específicamente en los siguientes aspectos: El Rostro de Jesús es una institución sin fines de lucro que al igual que la propuesta antropológica de Ricoeur, lo que se persigue es la recuperación del sujeto, en este sentido el filósofo francés aporta una base teórica muy interesante para definir el carácter institucional que busca la ipseidad de los internos. En este sentido,

por institución se debe entender, la estructura del vivir juntos de una comunidad histórica, estructura irreductible a las relaciones interpersonales y, sin embargo, unida a ellas en un sentido importante, que la noción de distribución permitirá 
aclarar después. La idea de institución se caracteriza fundamentalmente por costumbres comunes y no por reglas coaccionantes. (Ricoeur, 2003, p. 203)

Esta definición de institución está marcada por la idea del otro, es importante en la comunidad terapéutica del Rostro de Jesús. El otro es punto de referencia de la institución con el propósito de desarrollar el buen vivir de los seres humanos. (Ricoeur, 2003). Otro gran aporte de Sí Mismo como Otro de Paul Ricoeur, es el poder encontrar en esta obra, la práctica que se realiza en la CT Rostro de Jesús de buscar la igualdad a partir de las diferencias. La búsqueda de la igualdad a través de la desigualdad manifiesta la estima de sí entendida como el momento reflexivo del deseo de vida buena. Esto servirá para mejorar el concepto de comunidad terapéutica a partir de entender la identidad de uno en los otros, incorporando para ello la solicitud como estima del otro por mí mismo (Ricoeur, 2003).

Uno de los mensajes que se le da al adicto en la CT, Rostro de Jesús es la importancia de recuperar la estima de sí. Sí Mismo como Otro, expone el sentido existencial de la recuperación de la autoestima:

...no puedo estimarme a mí mismo sin estimar al otro como a mí mismo. Como a mí mismo significa: tú eres capaz de comenzar algo en el mundo, de actuar por razones, de jerarquizar tus preferencias, de estimar los fines de tu acción y, de este modo estimarte a ti miso como yo me estimo a mí mismo. La equivalencia entre tú también y el como a mí mismo descansa en una confianza que podemos considerar como una extensión de la atestación en virtud de la cual creo que yo puedo y valgo. (Ricoeur, 2003, p. 202)

Otro aporte importante de Sí Mismo como Otro a la CT, del Rostro de Jesús, corresponde a la dimensión ética, la atención que se ofrece debe fortalecer el deseo de una vida buena, sin quedarse solamente el eliminar el consumo de drogas. Aquí se encuentra el sustento filosófico de la premisa que el problema son las personas no las drogas. La ética de la institución no es cumplir, sino educar para una buena en todas sus dimensiones.

El aprecio por sí mismo y por demás es un deseo que en la CT quiere lograr con los internos que en ella se reciben, Sí Mismo como Otro, lo expone de forma magistral: Pues el sí mismo que uno ama es lo mejor de sí, llamado varias veces pensamiento o intelecto o incluso alma ...el bien más grande que el amigo desea a su amigo es que siga siendo lo que es" (Ricoeur, 2003, p. 191). 
En la CT, Rostro de Jesús, se expone a los internos que la recuperación de su ser persona depende mucho de la toma de conciencia de su problemática adictiva, Paul Ricoeur, amplia esta toma de conciencia y sin duda alguna es un gran aporte a la CT que se menciona en esta investigación:

La conciencia no es sólo conciencia de la percepción y de la actividad, sino también conciencia de vida. Por lo tanto en la medida que es agradable la conciencia de la vida, podemos decir que el sentido profundo de la philautía es deseo: la propia existencia del hombre de bien es apetecible para él (Ricoeur, 2003, p. 193).

La CT, considera que la adicción es una enfermedad severa y crónica, que trae consigo el sufrimiento y en esto consiste la rehabilitación, dar poder a la personas para que puedan hacer y ser alguien en la vida. En este sentido Sí Mismo como Otro expone un concepto de sufrimiento que es de suma importancia tomar en cuenta para la atención:

no se define únicamente por el dolor físico, ni siquiera por el dolor mental, sino por la disminución, incluso la destrucción de la capacidad de obrar, de poder hacer, parece incumbir exclusivamente al sí que da su simpatía, su compasión: estos términos se toman en el sentido de compartir la penas de otro. (p. 193).

La CT, desarrollada por el Rostro de Jesús, busca afectar la persona del adicto, se entiende esto, como la forma de influir en la vida de los internos. El aporte que la obra filosófica Sí Mismo como Otro, da a este aspecto del tratamiento, resume la razón de ser de la institución como comunidad de personas que quieren recuperarse. "La afección del sí por el otro distinto de sí, es el soporte de este intercambio regulado entre personas gramaticales” (Ricoeur, 2003, p. 366). El Rostro de Jesús transforma la relación yo- tú en sentido comunitario donde la identidad de la persona se refiere a sus ser comunitario, es allí donde se da la mayor afectación. Por eso el Rostro de Jesús al igual que lo que Planes Ricoeur, no concibe una buena vida con y para el otro en instituciones justas, sin la afección del proyecto de vivir bien, mediante la solicitud a la vez ejercida y recibida (Ricoeur, 2003, p. 366). El siguiente texto sustraído de Sí Mismo como otro resume e identifica el trabajo realizado en favor del adicto en la CT, Rostro de Jesús:

...la dialéctica de la estima de sí y de la amistad, antes incluso de cualquier consideración que descansa en la justicia de los intercambios, puede reescribirse totalmente en los términos de una dialéctica de la acción y de la afección... En este sentido, la amistad crea el hecho de la justicia, en cuanto virtud para otro...El obrar y el padecer parecen distribuidos así entre dos protagonistas diferentes; el agente y 
el paciente, éste último aparece como la víctima potencial del primero. (Ricoeur, 2003, p. 366-367).

Se estable de esta forma que en la relación de un operador de la CT con un paciente que recibe un tratamiento se establece un vínculo de identidad en donde cada uno desde su función, como paciente o como servidor de la CT, descubre su sí mismo en el otro.

\section{Conclusiones}

La Comunidad Terapéutica (CT), que se desarrolla, como forma atención al adicto a las drogas en el Centro de Rehabilitación al Adicto Rostro de Jesús, considera que dicha adicción es adherirse algo al propio ser ocultando la identidad de sí mismo sin que esta se pierda, por tal razón la obra Sí Mismo como Otro de Paul Ricouer, puede considerarse como referente teórico del programa de atención, pues tanto el cetro de Rehabilitación como la obra filosófica en cuestión, buscan como fin último la recuperación del sujeto. A través de la hermenéutica del sí, que es pensar en la identidad de sí mismo como otro, el abordaje de la adicción en la CT, podría tener un mejor sentido de interpretación de la vida de las personas.

Sí Mismo como Otro, le da un mejor sentido terapéutico a la atención ofrecida en la CT del Rostro de Jesús, puesto que las preguntas ¿quién soy?, ¿quién es el que habla?, ¿quién es el que actúa?, ¿quién es el otro?, no se responderían desde frases ya predeterminadas, sino utilizando el rodeo de la hermenéutica de Ricoeur, que se centra el ser de la persona y no en el cumplimiento del tiempo que dure el programa. Estas preguntas se convertirían en aprendizaje permanentes con el fin de llevar al paciente a una buena vida entendida como el poder de hacer algo, de esta forma la responsabilidad consistiría en capacidad para responder y no en reconocer que se consume una droga. Esto permitiría definir el concepto de rehabilitación como habilitar para. Se dejaría la tendencia de creer que ser rehabilitado es dejar el consumo nada más.

Los miembros que conforman la CT, en especial los pacientes, narran historias de vida que parecen fantasías o situaciones que no podría creerse. Sí Mismo como Otro, aporta a que cuando una persona habla, cuando dice algo, dice algo de sí. De esta manera, se propone un cambio en el abordaje del paciente, más que darle consejos y devolver lo que se interpreta, es descubrir qué identidad se manifiesta en ese relato. Por eso es importante valorar que entre más hable un paciente más expone su relación con los demás. La identidad narrativa de la que habla Ricouer, es uno de 
InterSedes, N44. Vol XXI (2020). ISSN 2215-2458

los principios que el Rostro de Jesús podría incorporar para mejorar su programa de atención y formar una actitud terapéutica.

La recuperación de la persona, tema de Sí Mismo como Otro y de la CT, Rostro de Jesús, parte del reconocimiento de sí mismo a partir del aprendizaje de las experiencias por muy doloras que sean. El estudio noveno, el sí y la sabiduría práctica: la convicción, de Sí Mismo como Otro, constituye un gran aporte para la CT, pues allí se encuentra el sustento teórico para verificar el cambio de vida que va realizando la persona desde el inicio hasta el final del internamiento. 
InterSedes, N44. Vol XXI (2020). ISSN 2215-2458

\section{Bibliografía}

Cuenca, A. (2012). Rastros antropológico-ético en el pensamiento de Paul Ricouer http://digitum.um.es/xmlui/bitstream/10201/10233/1/Rasgos\%20antropologicoeticos\%20en\%20e $1 \% 20$ pensamiento.pdf

Escohotado, A. (2008). Historia General de las Drogas. Madrid: Alianza Editorial.

Fernández, J. (2007) Propuestas Antropológicas del siglo XX (II). Pamplona. EUNSA

Gevaert, J. (2005) El problema del hombre: introducción a la antropología filosófica (14 ${ }^{\mathrm{a}}$ ed.). Salamanca, ES.: Ediciones Sígueme.

Mora, G. (2017). Programa de atención al adicto, Rostro de Jesús. Alajuela. Sin publicar.

Moizeszowicz, J. (2000). Psicofarmacología psicodinámica IV: estrategias terapéuticas y psiconeurobiológicas. Buenos Aires: Paidós.

Ricoeur, P. (1990). Historia y Verdad. (2a ed) Madrid.: Encuentro

. (2003) Sí Mismo como Otro (2 ed). Buenos Aíres.: SIGLO XXI

(2011) Finitud y culpabilidad ( $2^{\mathrm{a}}$ ed). Madrid.: Trotta.

Triana, M. (1997). Obligación y compromiso, estudio sobre la ética en el pensamiento filosófico de Paul Ricouer. Tesis sometida a la consideración del programa de estudios de posgrado en filosofía para optar por el grado de doctor en filosofía. San José. Universidad de Costa Rica. 